\title{
Evaluating heart transplantation outcomes from marginal donors
} \author{
Atieh Rezai ${ }^{4}$, AmirAli Hamidieh ${ }^{6}$, Marzieh Latifi ${ }^{1}$ \\ ${ }^{1}$ Department of Cardiology, Sina Hospital, Tehran University of Medical Sciences, Tehran, Iran \\ ${ }^{2}$ Department of Cardiovascular Surgery, Sina Hospital, Tehran University of Medical Sciences, Tehran, Iran \\ ${ }^{3}$ Cardiac Primary Prevention Research Center, Sina Hospital, Tehran University of Medical Sciences, Tehran, Iran \\ ${ }^{4}$ Department of Cardiology, Sina Hospital, Tehran University of Medical Sciences, Tehran, Iran \\ ${ }^{5}$ Sina Hospital, Tehran University of Medical Sciences, Tehran, Iran \\ ${ }^{6}$ Iranian Tissue Bank \& Research Center, Tehran University of Medical Sciences, Tehran, Iran
}

Azadeh Sadat Naseri ${ }^{1}$, Behnam Shakerian ${ }^{2}$, Farzaneh Bagherpour ${ }^{1}$, Haleh Ashraf ${ }^{3}$, Shahrokh Karbalai ${ }^{4}$, Abbas Soleimani ${ }^{5}$,

Background: Considering lack of enough resources, such as artificial heart or ventricular assist devices for long term cardiac replacement therapy, we decided to evaluate those brain death cases, which seem non eligible as heart donor based on guidelines criteria, as marginal donners, but with no contraindication for replacement at preoperational evaluation.

Methods: This retrospective study was conducted on heart donors and their recipients at Organ Procurement Unit of Sina. Among the candidates, 75 were categorized as standard donors (group A) and 18 were marginal donors (group B), group C were heart recipients from standard donors, and group $D$ were heart recipients from marginal donors.

Results: Based on this study 97 heart donors of a total number of 302 donors referred to Sina Hospital, $80.6 \%$ were sub grouped as group A, standard donor, and $19.4 \%$ group B as marginal group (older than 40 years, or with positive history of drug abuse or smoking, but based on echocardiography and coronary angiography, negative for HIV, or hepatitis infection). Their mean survival rate in groups $C$ and $D$ were $635.67 \pm 434.75$ and $508.46 \pm 407.8$ days respectively with no significant difference between survival rates in MD and SD recipients $(P=0.961)$.

Conclusions: Based on this study, marginal donors could be eligible for harvesting, and decrease wait time for end stage heart recipients.

Corresponding author: Sanaz Dehghani

E-mail: sanaz_dehghani2002@yahoo.com

\section{(c) The Korean Society for Transplantation}

This is an Open Access article distributed under the terms of the Creative Commons Attribution Non-Commercial License (http://creativecommons.org/licenses/by-nc/4.0/) which permits unrestricted non-commercial use, distribution, and reproduction in any medium, provided the original work is properly cited. 